\title{
Couplings of Pions with Excited Heavy Mesons From Light-Cone QCD Sum Rules in the Leading Order of HQET
}

\author{
Yuan-Ben Dai and Shi-Lin Zhu \\ Institute of Theoretical Physics, Academia Sinica, P.O.Box 2735, Beijing 100080, China
}

\begin{abstract}
The couplings of pions with lowest three doublets $\left(0^{-}, 1^{-}\right),\left(0^{+}, 1^{+}\right)$and $\left(1^{+}, 2^{+}\right)$ of heavy mesons are studied with light-cone QCD sum rules in the leading order of heavy quark effective theory. The ambiguity due to presence of two distinct $1^{+}$states are solved.
\end{abstract}

PACS number: 12.39.Hg, 14.40.Nd, 12.38.Lg

\section{Introduction}

Remarkbale progress has been made in understanding the physics of heavy mesons composed of a heavy quark and a light quark with the discovery of the heavy quark symmetry. To obtain detailed predictions one has to employ some specific nonperturbative methods, among which QCD sum rules [1] is very useful. The heavy quark effective theory (HQET) (2) provides a systematic expansion of QCD in terms of $1 / m_{Q}$, where $m_{Q}$ is the heavy quark mass. The spectrum of the ground state heavy meson has been studied with the QCD sum rules in HQET in [3]. In [⿴囗十 the mass of the lowest excited heavy meson doublets $\left(2^{+}, 1^{+}\right)$and $\left(1^{+}, 0^{+}\right)$were studied with QCD sum rules in the heavy quark effective theory (HQET) up to the order of $\mathcal{O}\left(1 / m_{Q}\right)$.

QCD sum rules has been used to analyse the couplings of the heavy hadrons with pions [5-15]. The widths for pionic decays of the lowest two excited doublets $\left(2^{+}, 1^{+}\right)$and $\left(1^{+}, 0^{+}\right)$is calculated with conventional QCD sum rules in the leading order of HQET in [5]. Light-cone QCD sum rules (LCQSR) with the full QCD Lagrangian is first employed to derive the strong coupling constants: $g_{\pi D^{*} D}, g_{\pi B^{*} B}$ [11]. Recently the strong coupling constants: $g_{\pi B^{*} B^{*}}, g_{\pi B_{1}^{\prime} B_{0}^{\prime}}$ and $g_{\pi B_{1}^{\prime} B^{*}}$ were calculated using LCQSR with finite heavy quark mass [13]. The couplings of heavy baryons with soft pions have been estimated with QCD sum rules in an external axial field [14. In this work we employ the LCQSR in HQET to calculate the on-shell couplings of the pions with heavy meson doublets $\left(0^{-}, 1^{-}\right),\left(0^{+}, 1^{+}\right)$ and $\left(1^{+}, 2^{+}\right)$to the leading order of $1 / m_{Q}$.

The LCQSR is quite different from the conventional QCD sum rules, which is based on the short-distance operator product expansion (OPE). The LCQSR is based on the 
OPE on the light cone, which is the expansion over the twists of the operators. The main contribution comes from the lowest twist operator. Matrix elements of nonlocal operators sandwiched between a hadronic state and the vacuum defines the hadron wave functions. When the LCQSR is used to calculate the coupling constant, the double Borel transformation is always invoked so that the excited states and the continuum contribution can be treated quite nicely. Moreover, the final sum rule depends only on the value of the wave function at a specific point like $\varphi_{\pi}\left(u_{0}=\frac{1}{2}\right)$, which is much better known than the whole wave function [11].

One difficult problem encountered in studying the decay widths of excited heavy mesons with QCD sum rules is the following. Except for the lowest states $0^{-}, 1^{-}$, the spectra contains a pair of states for any spin-parity $j^{P}$ with close values in their masses but quite different in magnitudes of their decay widths. In our case, one of the two $1^{+}$states is a narrow resonance decaying mainly by emitting a $D$ wave pion, while the other one is a very wide resonance decaying by emitting a $S$ wave pion. An interpolating current used for the narrow $1^{+}$state with a small coupling to the other $1^{+}$state may cause sizable error in the result of calculation. It is only in the $m_{Q} \rightarrow \infty$ limit, there is a conserved quantum number $j_{\ell}$, the angular momentum of the light component, which can be used to differentiate the two states. Therefore, HQET has important and unique advantage for this purpose. These are the motivation for our approach of using LCQSR in HQET.

The proper interpolating current $J_{j, P, j_{\ell}}^{\alpha_{1} \cdots \alpha_{j}}$ for the states with the quantum number $j, P$, $j_{\ell}$ in HQET was given in [4]. They were proved to satisfy the following conditions

$$
\begin{aligned}
\left\langle 0\left|J_{j, P, j_{\ell}}^{\alpha_{1} \cdots \alpha_{j}}(0)\right| j^{\prime}, P^{\prime}, j_{\ell}^{\prime}\right\rangle= & f_{P j_{l}} \delta_{j j^{\prime}} \delta_{P P^{\prime}} \delta_{j \ell j_{\ell}} \eta^{\alpha_{1} \cdots \alpha_{j}} \\
i\left\langle 0\left|T\left(J_{j, P, j_{\ell}}^{\alpha_{1} \cdots \alpha_{j}}(x) J_{j^{\prime}, P^{\prime}, j_{\ell}^{\prime}}^{\beta_{1} \cdots \beta_{j^{\prime}}}(0)\right)\right| 0\right\rangle= & \delta_{j j^{\prime}} \delta_{P P^{\prime}} \delta_{j \ell j_{\ell}^{\prime}}(-1)^{j} \mathcal{S} g_{t}^{\alpha_{1} \beta_{1}} \cdots g_{t}^{\alpha_{j} \beta_{j}} \\
& \times \int d t \delta(x-v t) \Pi_{P, j_{\ell}}(x)
\end{aligned}
$$

in the $m_{Q} \rightarrow \infty$ limit, where $\eta^{\alpha_{1} \cdots \alpha_{j}}$ is the polarization tensor for the spin $j$ state, $v$ is the velocity of the heavy quark, $g_{t}^{\alpha \beta}=g^{\alpha \beta}-v^{\alpha} v^{\beta}$ is the transverse metric tensor, $\mathcal{S}$ denotes symmetrizing the indices and subtracting the trace terms separately in the sets $\left(\alpha_{1} \cdots \alpha_{j}\right)$ and $\left(\beta_{1} \cdots \beta_{j}\right), f_{P, j_{\ell}}$ and $\Pi_{P, j_{\ell}}$ are a constant and a function of $x$ respectively which depend only on $P$ and $j_{\ell}$. Because of equations (1) and (2), the sum rule in HQET for decay widths derived from a correlator containing such currents receive no contribution from the unwanted states with the same spin-parity as the states under consideration in the $m_{Q} \rightarrow \infty$. Starting from the calculations in the leading order, the decay amplitudes for finite $m_{Q}$ can be calculated unambiguously order by order in the $1 / m_{Q}$ expansion in HQET.

\section{Sum rules for decay amplitudes}

In the present work we shall confine ourselves to the lowest lying states in the leading order of $1 / m_{Q}$ expansion. Denote the doublet $\left(1^{+}, 2^{+}\right)$with $j_{\ell}=3 / 2$ by $\left(B_{1}, B_{2}^{*}\right)$ and the doublet $\left(0^{+}, 1^{+}\right)$with $j_{\ell}=1 / 2$ by $\left(B_{0}^{\prime}, B_{1}^{\prime}\right)$. There are many combinations for the coupling constant $g_{\pi B_{x} B_{y}}$, where $B_{x}, B_{y}$ belongs to the three doublets $\left(0^{-}, 1^{-}\right),\left(0^{+}, 1^{+}\right)$ 
and $\left(1^{+}, 2^{+}\right)$. Due to the heavy quark symmetry and chiral symmetry there exist only six independent coupling constants, i.e., three independent coupling constants when $B_{x}, B_{y}$ belongs to different doublets and another three when $B_{x}, B_{y}$ belongs to the same doublet. For example, from covariance and conservation of the angular momentum of the light component in the $m_{Q} \rightarrow \infty$ limit, the amplitudes for the decay of $B_{1}, B_{2}^{*}$ to the ground states $B, B^{*}$ in the doublet $\left(0^{-}, 1^{-}\right)$with $j_{\ell}=1 / 2$ have the following forms

$$
\begin{aligned}
M\left(B_{1} \rightarrow B^{*} \pi\right) & =I \epsilon_{\mu}^{*} \eta_{\nu}\left(q_{t}^{\mu} q_{t}^{\nu}-\frac{1}{3} g_{t}^{\mu \nu} q_{t}^{2}\right) g\left(B_{1}, B^{*}\right), \\
M\left(B_{2}^{*} \rightarrow B \pi\right) & =I \eta_{\mu \nu} q_{t}^{\mu} q_{t}^{\nu} g\left(B_{2}^{*}, B\right), \\
M\left(B_{2}^{*} \rightarrow B^{*} \pi\right) & =I i \varepsilon_{\alpha \beta \sigma \rho} \epsilon^{* \alpha} v^{\beta} \eta^{\sigma \mu} q_{t}^{\rho} q_{t \mu} g\left(B_{2}^{*}, B^{*}\right),
\end{aligned}
$$

where $\eta_{\mu \nu}, \eta_{\mu}$ and $\epsilon_{\mu}$ are polarization tensors for states $2^{+}, 1^{+}$and $1^{-}$respectively. $q_{t \mu}=$ $q_{\mu}-v \cdot q v_{\mu} . I=\sqrt{2}, 1$ for charged and neutral pion respectively. As shown in [15] the constants in (3)-(5) satisfy

$$
g\left(B_{2}^{*}, B\right)=g\left(B_{2}^{*}, B^{*}\right)=\sqrt{\frac{2}{3}} g\left(B_{1}, B^{*}\right) .
$$

We calculate the following six independent coupling constants: $g_{1}=g_{\pi B_{1} B^{*}}, g_{2}=g_{\pi B_{0}^{\prime} B}$, $g_{3}=g_{\pi B_{1}^{\prime} B_{0}^{\prime}}, g_{4}=g_{\pi B_{2}^{*} B_{1}}, g_{5}=g_{\pi B_{1}^{\prime} B_{1}}$ and $g_{6}=g_{\pi B^{*} B}$. Other coupling constants are related to them. There are related results for $g_{2}, g_{3}$ and $g_{6}$ in [6-15]. In [5] $g_{1}$ and $g_{2}$ is calculated with QCD sum rules in the short distance expansion. For the sake of comparison and completeness, we also present the sum rules for $g_{2}, g_{3}$ and $g_{6}$.

For deriving the sum rules for the coupling constants we consider the correlators

$$
\begin{array}{r}
\int d^{4} x e^{-i k \cdot x}\left\langle\pi(q)\left|T\left(J_{1,-, \frac{1}{2}}^{\beta}(0) J_{1,+, \frac{3}{2}}^{\dagger \alpha}(x)\right)\right| 0\right\rangle=\left(q_{t}^{\alpha} q_{t}^{\beta}-\frac{1}{3} g_{t}^{\alpha \beta} q_{t}^{2}\right) I G_{B_{1} B^{*}}\left(\omega, \omega^{\prime}\right) \\
\int d^{4} x e^{-i k \cdot x}\left\langle\pi(q)\left|T\left(J_{0,+, \frac{1}{2}}(0) J_{0,-, \frac{1}{2}}^{\dagger}(x)\right)\right| 0\right\rangle=I G_{B_{0}^{\prime} B}\left(\omega, \omega^{\prime}\right) \\
\int d^{4} x e^{-i k \cdot x}\left\langle\pi(q)\left|T\left(J_{0,+, \frac{1}{2}}(0) J_{1,+, \frac{1}{2}}^{\dagger \alpha}(x)\right)\right| 0\right\rangle=q_{t}^{\alpha} I G_{B_{1}^{\prime} B_{0}^{\prime}}\left(\omega, \omega^{\prime}\right), \\
\int d^{4} x e^{-i k \cdot x}\left\langle\pi(q)\left|T\left(J_{1,+, \frac{3}{2}}^{\alpha}(0) J_{2,+, \frac{3}{2}}^{\dagger \sigma \beta}(x)\right)\right| 0\right\rangle= \\
\left.\int q_{t}^{\alpha} q_{t}^{\sigma} q_{t}^{\beta}-\frac{1}{6} q_{t}^{2}\left(g_{t}^{\alpha \beta} q_{t}^{\sigma}+g_{t}^{\alpha \sigma} q_{t}^{\beta}+\frac{4}{3} g_{t}^{\sigma \beta} q_{t}^{\alpha}\right)\right) I G_{B_{2}^{*} B_{1}}\left(\omega, \omega^{\prime}\right) \\
\int d^{4} x e^{-i k \cdot x}\left\langle\pi(q)\left|T\left(J_{1,+, \frac{3}{2}}^{\alpha}(0) J_{1,+, \frac{1}{2}}^{\dagger \beta}(x)\right)\right| 0\right\rangle=i \epsilon^{\alpha \beta \sigma \delta} q_{\sigma}^{t} v_{\delta} I G_{B_{1}^{\prime} B_{1}}\left(\omega, \omega^{\prime}\right) \\
\int d^{4 k \cdot x}\left\langle\pi(q)\left|T\left(J_{1,-, \frac{1}{2}}^{\alpha}(0) J_{0,-, \frac{1}{2}}^{\dagger}(x)\right)\right| 0\right\rangle=q_{t}^{\alpha} I G_{B^{*} B}\left(\omega, \omega^{\prime}\right),
\end{array}
$$

where $k^{\prime}=k+q, \omega=2 v \cdot k, \omega^{\prime}=2 v \cdot k^{\prime}$ and $q^{2}=0$. The forms of the right hand side of (7)-(12) are determined by the conservation of angular momentum of the light component and the fact that $\alpha, \beta, \sigma$ and $\delta$ are transverse indices, $x-y=v t$ on the heavy quark 
propagator. The interpolationg currents are given in [4] as

$$
\begin{aligned}
J_{1,+, \frac{3}{2}}^{\dagger \alpha} & =\sqrt{\frac{3}{4}} \bar{h}_{v} \gamma^{5}(-i)\left(\mathcal{D}_{t}^{\alpha}-\frac{1}{3} \gamma_{t}^{\alpha} \mathcal{D}_{t}\right) q, \\
J_{2,+, \frac{3}{2}}^{\dagger \alpha_{1}, \alpha_{2}} & =\sqrt{\frac{1}{2}} \bar{h}_{v} \frac{(-i)}{2}\left(\gamma_{t}^{\alpha_{1}} \mathcal{D}_{t}^{\alpha_{2}}+\gamma_{t}^{\alpha_{2}} \mathcal{D}_{t}^{\alpha_{1}}-\frac{2}{3} g_{t}^{\alpha_{1} \alpha_{2}} \mathcal{D}_{t}\right) q, \\
J_{1,-, \frac{1}{2}}^{\dagger \alpha} & =\sqrt{\frac{1}{2}} \bar{h}_{v} \gamma_{t}^{\alpha} q, \quad J_{0,-, \frac{1}{2}}^{\dagger \alpha}=\sqrt{\frac{1}{2}} \bar{h}_{v} \gamma_{5} q, \\
J_{0,+, \frac{1}{2}}^{\dagger} & =\frac{1}{\sqrt{2}} \bar{h}_{v} q, \quad J_{1,+, \frac{1}{2}}^{\dagger \alpha}=\frac{1}{\sqrt{2}} \bar{h}_{v} \gamma^{5} \gamma_{t}^{\alpha} q .
\end{aligned}
$$

where $h_{v}$ is the heavy quark field in HQET and $\gamma_{t \mu}=\gamma_{\mu}-v_{\mu} \psi$.

Let us first consider the function $G_{B_{1} B^{*}}\left(\omega, \omega^{\prime}\right)$ in (7). As a function of two variables, it has the following pole terms from double dispersion relation

$$
\frac{f_{-, \frac{1}{2}} f_{+, \frac{3}{2}} g\left(B_{1} B^{*}\right)}{\left(2 \bar{\Lambda}_{-, \frac{1}{2}}-\omega^{\prime}\right)\left(2 \bar{\Lambda}_{+, \frac{3}{2}}-\omega\right)}+\frac{c}{2 \bar{\Lambda}_{-, \frac{1}{2}}-\omega^{\prime}}+\frac{c^{\prime}}{2 \bar{\Lambda}_{+, \frac{3}{2}}-\omega},
$$

where $f_{P, j_{\ell}}$ are constants defined in (1), $\bar{\Lambda}_{P, j_{\ell}}=m_{P, j_{\ell}}-m_{Q}$. As explained in Section 1, only one state with $j^{P}=1^{+}$contributes to (17) as the result of equation (11). This would not be true if the last term in (13) is absent.

For deriving QCD sum rules we calculate the correlator (7) by the operator expansion on the light-cone in HQET to the leading order of $\mathcal{O}\left(1 / m_{Q}\right)$. The expression for $G_{B_{1} B^{*}}\left(\omega, \omega^{\prime}\right)$ reads

$$
\frac{\sqrt{6}}{8} \int_{0}^{\infty} d t \int d x e^{-i k x} \delta(-x-v t) \operatorname{Tr}\left\{\gamma_{\beta}^{t}(1+\hat{v}) \gamma_{5}\left(D_{\alpha}^{t}-\frac{1}{3} \gamma_{\alpha}^{t} \hat{D}^{t}\right)\langle\pi(q)|u(0) \bar{d}(x)| 0\rangle\right\}
$$

The pion wave function is defined as the matrix elements of nonlocal operators between the vacuum and pion state. Up to twist four they are [11]:

$$
\begin{aligned}
<\pi(q)\left|\bar{d}(x) \gamma_{\mu} \gamma_{5} u(0)\right| 0> & =-i f_{\pi} q_{\mu} \int_{0}^{1} d u e^{i u q x}\left(\varphi_{\pi}(u)+x^{2} g_{1}(u)+\mathcal{O}\left(x^{4}\right)\right) \\
& +f_{\pi}\left(x_{\mu}-\frac{x^{2} q_{\mu}}{q x}\right) \int_{0}^{1} d u e^{i u q x} g_{2}(u) \\
<\pi(q)\left|\bar{d}(x) i \gamma_{5} u(0)\right| 0> & =\frac{f_{\pi} m_{\pi}^{2}}{m_{u}+m_{d}} \int_{0}^{1} d u e^{i u q x} \varphi_{P}(u) \\
<\pi(q)\left|\bar{d}(x) \sigma_{\mu \nu} \gamma_{5} u(0)\right| 0> & =i\left(q_{\mu} x_{\nu}-q_{\nu} x_{\mu}\right) \frac{f_{\pi} m_{\pi}^{2}}{6\left(m_{u}+m_{d}\right)} \int_{0}^{1} d u e^{i u q x} \varphi_{\sigma}(u) .
\end{aligned}
$$

The wave function $\varphi_{\pi}$ is associated with the leading twist two operator, $g_{1}$ and $g_{2}$ correspond to twist four operators, and $\varphi_{P}$ and $\varphi_{\sigma}$ to twist three ones. Due to the choice of the gauge $x^{\mu} A_{\mu}(x)=0$, the path-ordered gauge factor $P \exp \left(i g_{s} \int_{0}^{1} d u x^{\mu} A_{\mu}(u x)\right)$ has been omitted. 
Expressing (18) with the pion light-cone wave functions, we arrive at $G_{B_{1} B^{*}}\left(\omega, \omega^{\prime}\right)=-i \frac{\sqrt{6}}{8} F_{\pi} \int_{0}^{\infty} d t \int_{0}^{1} d u e^{i(1-u) \frac{\omega t}{2}} e^{i u \frac{\omega^{\prime} t}{2}} u\left\{\varphi_{\pi}(u)+t^{2} g_{1}(u)+\frac{i t}{q \cdot v} g_{2}(u)+\frac{i t}{6} \mu_{\pi} \varphi_{\sigma}(u)\right\}+\cdots$,

where $\mu_{\pi} \equiv \frac{m_{\pi}^{2}}{m_{u}+m_{d}}=1.76 \mathrm{GeV}, F_{\pi}=\frac{f_{\pi}}{\sqrt{2}}=92 \mathrm{MeV}$ for neutral pions. For large euclidean values of $\omega$ and $\omega^{\prime}$ this integral is dominated by the region of small $t$, therefore it can be approximated by the first a few terms.

Similarly, we have:

$$
\begin{gathered}
G_{B_{0}^{\prime} B}\left(\omega, \omega^{\prime}\right)=\frac{i}{4} F_{\pi} \int_{0}^{\infty} d t \int_{0}^{1} d u e^{i(1-u) \frac{\omega t}{2}} e^{i u \frac{\omega^{\prime} t}{2}}\left\{\mu_{\pi} \varphi_{P}(u)-(q \cdot v)\left[\varphi_{\pi}(u)+t^{2} g_{1}(u)\right]\right\} \\
G_{B_{1}^{\prime} B_{0}^{\prime}}\left(\omega, \omega^{\prime}\right)=\frac{i}{4} F_{\pi} \int_{0}^{\infty} d t \int_{0}^{1} d u e^{i(1-u) \frac{\omega t}{2}} e^{i u \frac{\omega^{\prime} t}{2}}\left\{\varphi_{\pi}(u)+t^{2} g_{1}(u)+\frac{i t}{q \cdot v} g_{2}(u)-\frac{i t}{6} \mu_{\pi} \varphi_{\sigma}(u)\right\} \\
G_{B_{2}^{*} B_{1}}\left(\omega, \omega^{\prime}\right)=-i \frac{\sqrt{6}}{8} F_{\pi} \int_{0}^{\infty} d t \int_{0}^{1} d u e^{i(1-u) \frac{\omega t}{2}} e^{i u \frac{\omega^{\prime} t}{2}} u^{2}\left\{\varphi_{\pi}(u)+t^{2} g_{1}(u)+\frac{i t}{q \cdot v} g_{2}(u)+\frac{i t}{6} \mu_{\pi} \varphi_{\sigma}(u)\right\} \\
G_{B_{1}^{\prime} B_{1}}\left(\omega, \omega^{\prime}\right)=i \frac{\sqrt{6}}{24} F_{\pi} \int_{0}^{\infty} d t \int_{0}^{1} d u e^{i(1-u) \frac{\omega t}{2}} e^{i u \frac{\omega^{\prime} t}{2}} u\left\{\mu_{\pi} \varphi_{P}(u)+(q \cdot v)\left[\varphi_{\pi}(u)+t^{2} g_{1}(u)\right]\right\} \\
G_{B^{*} B}\left(\omega, \omega^{\prime}\right)=\frac{i}{4} F_{\pi} \int_{0}^{\infty} d t \int_{0}^{1} d u e^{i(1-u) \frac{\omega t}{2}} e^{i u \frac{\omega^{\prime} t}{2}}\left\{\varphi_{\pi}(u)+t^{2} g_{1}(u)+\frac{i t}{q \cdot v} g_{2}(u)+\frac{i t}{6} \mu_{\pi} \varphi_{\sigma}(u)\right\}
\end{gathered}
$$

After performing Wick rotation and double Borel transformation with the variables $\omega$ and $\omega^{\prime}$ the single-pole terms in (17) are eliminated. Subtracting the continuum contribution which is modeled by the integral in region $\omega, \omega^{\prime} \geq \omega_{c}$, we arrive at:

$g_{1} f_{-, \frac{1}{2}} f_{+, \frac{3}{2}}=-\frac{\sqrt{6}}{4} F_{\pi} e^{\Lambda_{-, \frac{1}{2}}^{+\Lambda}+, \frac{3}{2}}\left\{u_{0} \varphi_{\pi}\left(u_{0}\right) T f_{0}\left(\frac{\omega_{c}}{T}\right)-\frac{4}{T} u_{0} g_{1}\left(u_{0}\right)+\frac{4}{T} G_{1}\left(u_{0}\right)+\frac{1}{3} \mu_{\pi} u_{0} \varphi_{\sigma}\left(u_{0}\right)\right\}$,

where $u_{0}=\frac{T_{1}}{T_{1}+T_{2}}, T=\frac{T_{1} T_{2}}{T_{1}+T_{2}}, T_{1}, T_{2}$ are the Borel parameters, $G_{1}\left(u_{0}\right) \equiv \int_{0}^{u_{0}} u g_{2}(u) d u$ and $f_{n}(x)=1-e^{-x} \sum_{k=0}^{n} \frac{x^{k}}{k !}$. The presence of the factor $f_{n}$ is the result of subtracting the integral $\int_{\omega_{c}}^{\infty} s^{n} e^{-\frac{s}{T}} d s$ as a contribution of the continum. In obtaining (28) we have used the Borel transformation formula: $\hat{\mathcal{B}}_{\omega}^{T} e^{\alpha \omega}=\delta\left(\alpha-\frac{1}{T}\right)$. The integral in the function $G_{1}\left(u_{0}\right)$ in (28) arises from the factor $1 /(q \cdot v)$ in (22). Here we have used integration by parts to absorb the factor $1 /(q \cdot v)$. In this way we arrive at the simple form after double Borel transformation. In the following we shall use the same technique to deal with the factor $(q \cdot v)^{-1}$ in other sum rules.

Similarly we have:

$$
g_{2} f_{-, \frac{1}{2}} f_{+, \frac{1}{2}}=\frac{1}{4} F_{\pi} e^{\Lambda_{-, \frac{1}{2}}^{+\Lambda_{+, \frac{1}{2}}}}\left\{-\varphi_{\pi}^{\prime}\left(u_{0}\right) T^{2} f_{1}\left(\frac{\omega_{c}}{T}\right)+2 \mu_{\pi} \varphi_{P}\left(u_{0}\right) T f_{0}\left(\frac{\omega_{c}}{T}\right)+4 g_{1}^{\prime}\left(u_{0}\right)\right\},
$$

where $\varphi_{\pi}^{\prime}\left(u_{0}\right), g_{1}^{\prime}\left(u_{0}\right)$ are the first derivatives of $\varphi_{\pi}(u), g_{1}(u)$ at $u=u_{0}$. The appearance of derivatives is due to the factor $(q \cdot v)$. 


$$
g_{3} f_{+, \frac{1}{2}}^{2}=\frac{1}{2} F_{\pi} e^{\frac{2 \Lambda_{+, \frac{1}{2}}}{T}}\left\{\varphi_{\pi}\left(u_{0}\right) T f_{0}\left(\frac{\omega_{c}}{T}\right)-\frac{4}{T} g_{1}\left(u_{0}\right)+\frac{4}{T} G_{3}\left(u_{0}\right)-\frac{1}{3} \mu_{\pi} \varphi_{\sigma}\left(u_{0}\right)\right\},
$$

where $G_{3}\left(u_{0}\right) \equiv \int_{0}^{u_{0}} g_{2}(u) d u$.

$$
g_{4} f_{+, \frac{3}{2}}^{2}=\frac{\sqrt{6}}{4} F_{\pi} e^{\frac{2 \Lambda_{+}, \frac{3}{2}}{T}}\left\{u_{0}^{2} \varphi_{\pi}\left(u_{0}\right) T f_{0}\left(\frac{\omega_{c}}{T}\right)-\frac{4}{T} u_{0}^{2} g_{1}\left(u_{0}\right)+\frac{4}{T} G_{4}\left(u_{0}\right)+\frac{1}{3} \mu_{\pi} u_{0}^{2} \varphi_{\sigma}\left(u_{0}\right)\right\}
$$

where $G_{4}\left(u_{0}\right) \equiv \int_{0}^{u_{0}} u^{2} g_{2}(u) d u$.

$$
\begin{gathered}
g_{5} f_{+, \frac{1}{2}} f_{+, \frac{3}{2}}=\frac{\sqrt{6}}{12} F_{\pi} e^{\frac{\Lambda_{+, \frac{1}{2}}+\Lambda_{+, \frac{3}{2}}}{T}}\left\{\left.\frac{d}{d u}\left(u \varphi_{\pi}(u)\right)\right|_{u_{0}} T^{2} f_{1}\left(\frac{\omega_{c}}{T}\right)+\mu_{\pi} u_{0} \varphi_{P}\left(u_{0}\right) T f_{0}\left(\frac{\omega_{c}}{T}\right)-\left.4 \frac{d}{d u}\left(u g_{1}(u)\right)\right|_{u_{0}}\right\} \\
g_{6} f_{-, \frac{1}{2}}^{2}=\frac{1}{2} F_{\pi} e^{\frac{2 \Lambda_{-, \frac{1}{2}}}{T}}\left\{\varphi_{\pi}\left(u_{0}\right) T f_{0}\left(\frac{\omega_{c}}{T}\right)-\frac{4}{T} g_{1}\left(u_{0}\right)+\frac{4}{T} G_{3}\left(u_{0}\right)+\frac{1}{3} \mu_{\pi} \varphi_{\sigma}\left(u_{0}\right)\right\}
\end{gathered}
$$

\section{Determination of the parameters}

In order to obtain the coupling constants from (28)-(33) we need to use the mass parameters $\bar{\Lambda}$ 's and the coupling constants $f$ 's of the corresponding interpolating currents as input. $\bar{\Lambda}_{-, 1 / 2}$ and $f_{-, 1 / 2}$ can be obtained from the results in [16] as $\bar{\Lambda}_{-, 1 / 2}=0.5 \mathrm{GeV}$ and $f_{-, 1 / 2} \simeq$ $0.25 \mathrm{GeV}^{3 / 2}$ at the order $\alpha_{s}=0$. Notice that the coupling constant $f_{-, 1 / 2}$ defined in the present work is a factor $1 / \sqrt{2}$ smaller than that defined in [16]. $\bar{\Lambda}_{+, 3 / 2}$ and $\bar{\Lambda}_{+, 1 / 2}$ are given in [4]. $f_{+, 3 / 2}$ and $f_{+, 1 / 2}$ can be determined from the formulas $(34),(27)$ and (28) of reference [4] derived from sum rules for two point correlators. The results are

$$
\begin{array}{ll}
\bar{\Lambda}_{+, 3 / 2}=0.82 \mathrm{GeV} & f_{+, 3 / 2}=0.19 \pm 0.03 \mathrm{GeV}^{5 / 2} \\
\bar{\Lambda}_{+, 1 / 2}=1.15 \mathrm{GeV} & f_{+, 1 / 2}=0.40 \pm 0.06 \mathrm{GeV}^{3 / 2}
\end{array}
$$

We use the wave functions adopted in [1] to compute the coupling constants. Moreover, we choose to work at the symmetric point $T_{1}=T_{2}=2 T$, i.e., $u_{0}=\frac{1}{2}$ as traditionally done in literature [11]. We adopt the scale $\mu=1.4 \mathrm{GeV}$, at which the values of the various functions appearing in (28)-(33), at $u_{0}=\frac{1}{2}$, are: $\varphi_{\pi}\left(u_{0}\right)=1.22 \pm 0.3, \varphi_{P}\left(u_{0}\right)=1.142, \varphi_{\sigma}\left(u_{0}\right)=1.463$, $g_{1}\left(u_{0}\right)=3.4 \times 10^{-2} \mathrm{GeV}^{2}, G_{1}\left(u_{0}\right)=-4.5 \times 10^{-3} \mathrm{GeV}^{2}, G_{3}\left(u_{0}\right)=-2.0 \times 10^{-2} \mathrm{GeV}^{2}$, $G_{4}\left(u_{0}\right)=-1.3 \times 10^{-3} \mathrm{GeV}^{2}, g_{2}\left(\frac{1}{2}\right)=0, \varphi_{\pi}^{\prime}\left(u_{0}\right)=0$ and $g_{1}^{\prime}\left(u_{0}\right)=0$

\section{Numerical results and discussion}

We now turn to the numerical evaluation of the sum rules for the coupling constants. The lower limit of $T$ is determined by the requirement that the terms of higher twists in the operator expansion is less than one third of the whole sum rule. This leads to $T>1.0 \mathrm{GeV}$ 
for the sum rules (28)-(33). In fact the twist-four terms contribute only a few percent to the sum rules for such $T$ values. The upper limit of $T$ is constrained by the requirement that the continuum contribution is less than $30 \%$. This corresponds to $T<2.5 \mathrm{GeV}$. With the values of pion wave functions at $u_{0}=\frac{1}{2}$ given above we obtain the left hand side of the sum rules (28)-(33) as functions of $T$. The results are shown in FIG. 1. Stability develops for the sum rule (28)-(33) in the region $1.0 \mathrm{GeV}<T<2.5 \mathrm{GeV}$. Numerically we have:

$$
\begin{aligned}
& g_{1} f_{-, \frac{1}{2}} f_{+, \frac{3}{2}}=-(0.16 \pm 0.02 \pm 0.03) \quad \mathrm{GeV}^{2}, \\
& g_{2} f_{-, \frac{1}{2}} f_{+, \frac{1}{2}}=(0.36 \pm 0.02 \pm 0.03) \quad \mathrm{GeV}^{3}, \\
& g_{3} f_{+, \frac{1}{2}}^{2}=(0.13 \pm 0.01 \pm 0.02) \quad \mathrm{GeV}^{2}, \\
& g_{4} f_{+, \frac{3}{2}}^{2}=(0.10 \pm 0.01 \pm 0.02) \quad \mathrm{GeV}^{2}, \\
& g_{5} f_{+, \frac{1}{2}} f_{+, \frac{3}{2}}=(0.20 \pm 0.01 \pm 0.02) \quad \mathrm{GeV}^{3} \\
& g_{6} f_{-, \frac{1}{2}}^{2}=(0.20 \pm 0.01 \pm 0.02) \quad \mathrm{GeV}^{2},
\end{aligned}
$$

where the first error refers to the variations with $T$ in this region and the second error takes into account the uncertaity in $\omega_{c}$. And the central value corresponds to $T=1.5 \mathrm{GeV}$ and $\omega_{c}=3.0 \mathrm{GeV}$ for $(28)-(32)$. For the sum rule (33) we use $\omega_{c}=2.4 \mathrm{GeV}$.

With the central values of $f$ 's in (34) we get the absolute value of the coupling constants:

$$
\begin{aligned}
& g_{1}=-(3.6 \pm 0.4 \pm 0.6) \quad \mathrm{GeV}^{-2}, \\
& g_{2}=3.6 \pm 0.3 \pm 0.6, \\
& g_{3}=0.83 \pm 0.1 \pm 0.2 \quad \mathrm{GeV}^{-1} \\
& g_{4}=2.7 \pm 0.2 \pm 0.5 \quad \mathrm{GeV}^{-3} \\
& g_{5}=2.6 \pm 0.2 \pm 0.4 \quad \mathrm{GeV}^{-1} \\
& g_{6}=3.1 \pm 0.3 \pm 0.6 \mathrm{GeV}^{-1}
\end{aligned}
$$

We have not included the uncertainties due to f's here. Note that the sum rules (28) and (30)-(33) are also sensitive to the value of $\varphi_{\pi}\left(\frac{1}{2}\right)$. For example, $g_{1}=-4.83$ with $\varphi_{\pi}\left(\frac{1}{2}\right)=1.71$ which is one of the values used in [21].

The couplings of pions with heavy mesons obey symmetry relations in the leading order of HQET. Only in HQET the ambiguity due to the presence of two distinct $1^{+}$states can be resolved. We use light-cone QCD sum rules to calculate all the six independent strong coupling constants $g_{1}-g_{6}$ for the lowest three doublets. The sum rules in HQET for $g_{3}$ and $g_{6}$ are consistent with those for $g$ and $g^{\prime}$ in [11, 13] in the limit of $m_{Q} \rightarrow \infty$ with $g_{3}=\frac{2 g^{\prime}}{f_{\pi}}$ and $g_{6}=\frac{2 g}{f_{\pi}}$. The sum rule (29) for $g_{2}$ can be compared to that for $h$ in 13 with $g_{2}=\frac{2 h}{f_{\pi}}$ from definition. In terms of $h$ our value is 0.25 , which is only one half of that given in 13. Our result $g_{1}=3.6$ is slightly smaller than that from the short-distance QCD sum rule [5]. The calculation for $g_{4}$ and $g_{5}$ is new.

With the CLEO measurement of $\mathcal{B}\left(D^{*+} \rightarrow D^{+} \gamma\right)$ [18], a model independent extraction of $g$ has been performed recently [19]. Two possible solutions of $g\left(g=\frac{f_{\pi}}{2} g_{6}\right.$ in our notation ) and $\beta$ are found from fitting to the experimental data, either $g=0.27_{-0.02-0.02}^{+0.04+0.03}$, $\beta=0.85_{-0.1-0.1}^{+0.2+0.3} \mathrm{GeV}^{-1}$ or $g=0.76_{-0.03}^{+0.03+0.1}, \beta=4.90_{-0.3-0.7}^{+0.3+5.0} \mathrm{GeV}^{-1}$. The $g=0.76$ solution is excluded by the experimental limit $\Gamma\left(D^{*+}\right)<0.13 \mathrm{MeV}$ [20]. The coupling $g$ from the 
LCQSR approach is $g \sim 0.21-0.40$ [7, 12, 11], which is consistent with the experimentally favored solution $g=0.27$. In a recent work [21] the mixing effect of the two $1^{+}$states in the lowest excited heavy meson doublets $\left(2^{+}, 1^{+}\right)$and $\left(1^{+}, 0^{+}\right)$and the $\mathcal{O}\left(1 / m_{Q}\right)$ correction to the leading order coupling $g_{1}$ was calculated. The resulting pionic decay widths of $D_{2}^{*}(2460)$ and $D_{1}(2420)$ are in agreement with the experimental data [22.

Acknowledgements: S.Z. was supported by the National Postdoctoral Science Foundation of China and Y.D. was supported by the National Natural Science Foundation of China.

\section{References}

[1] M.A. Shifman, A.I. Vainshtein and V.I. Zakharov, Nucl. Phys. B 174 (1979) 385, 448, 519 .

[2] B. Grinstein, Nucl. Phys. B339 (1990) 253; E. Eichten and B. Hill, Phys. Lett. B234 (1990) 511; A. F. Falk, H. Georgi, B. Grinstein and M. B. Wise, Nucl. Phys. B343 (1990) 1; F. Hussain, J. G. Körner, K. Schilcher, G. Thompson and Y. L. Wu, Phys. Lett. B249 (1990) 295; J. G. Körner and G. Thompson, Phys. Lett. B264 (1991) 185.

[3] P. Colangelo, G. Nardulli, A. A. Ovchinnikov and N. Paver, Phys. Lett. B269 (1991) 204; P. Colangelo, G. Nardulli and N. Paver, Phys. Lett. B293 (1992) 207; P. Colangelo, F. De Fazio, G. Nardulli, N. Di Bartolomeo and R. Gatto, Phys. Rev. D 52 (1995) 6422.

[4] Y. B. Dai, C. S. Huang, M. Q. Huang and C. Liu, Phys. Lett. B390 (1997) 350; Y. B. Dai, C. S. Huang and M. Q. Huang, Phys. Rev. D 55 (1997) 5719.

[5] Y.B.Dai et al., report BIHEP-TH-97-005 (hep-ph/9705223).

[6] N. Di Bartolomeo, F. Feruglio, R. Gatto and G. Nardulli, Phys. Lett. B 347 (1995) 405.

[7] P. Colangelo, G.Nardulli, A.Deandrea, N.Di Bartolomeo, R.Gatto and F.Feruglio, Phys. Lett. B 339 (1994) 151.

[8] V.L.Eletski and Y.I.Kogan, Z.Phys. C 28 (1985) 155.

[9] A.G.Grozin and O.I.Yakovlev, preprint BUDKERINP-94-3 (hep-ph/9401267).

[10] S.Narison and H.G.Dosch, Phys. Lett. B 368 (1996) 163.

[11] V.M.Belyaev, V.M.Braun, A.Khodjamirian and R.Rückl, Phys. Rev. D 51 (1995) 6177. 
[12] T.M. Aliev, D.D. Demir, E. Iltan, and N.K. pak, Phys. Lett. B351 (1995) 339; T.M. Aliev, N.K. Pak and M.Savci, Phys. Lett. B 390 (1997) 335.

[13] P. Colangelo and F. de Fazio, BARI-TH/97-250 (hep-ph/9706271).

[14] A. G. Grozin and O. I. Yakovlev, WUE/ITP-97-016 (hep-ph/9706421).

[15] A. Falk and M. Luke, Phys. Lett. B292 (1992) 119; U. Kilian, J.G. Körner, and D. Pirjol, Phys. Lett. B288 (1992) 360.

[16] E. Bagan, P. Ball, V. M. Braun and H. G. Dosch, Phys. Lett. B278 (1992) 457; M. Neubert, Phys. Rev. D 45 (1992) 2451; D. J. Broadhurst and A. G. Grozin, Phys. Lett. B274 (1992) 421.

[17] Y. B. Dai and H. Y. Jin, Phys. Rev. D 52 (1995) 236.

[18] CLEO Collaboration, hep-ex/9711011.

[19] I. W. Stewart, hep-ph/9803227.

[20] ACCMOR Collaboration, Phys. Lett. B278 (1992) 480.

[21] Yuan-Ben Dai and Shi-Lin Zhu, hep-ph/9802224.

[22] Particle Data Group, R. M. Barnett et. al., Phys. Rev. D 54 (1996) 1.

\section{Figure Captions}

FIG. 1. Dependence of $f_{i} f_{j} g_{1-6}$ in (28)-(33) on the Borel parameter $T$ for the continuum threshold $\omega_{c}=3.0 \mathrm{GeV}$. The dotted, solid, short-dashed, long-dashed, dot-dashed and intermediate-dashed curves correspond to the coupling constant $g_{1}-g_{6}$ respectively. 


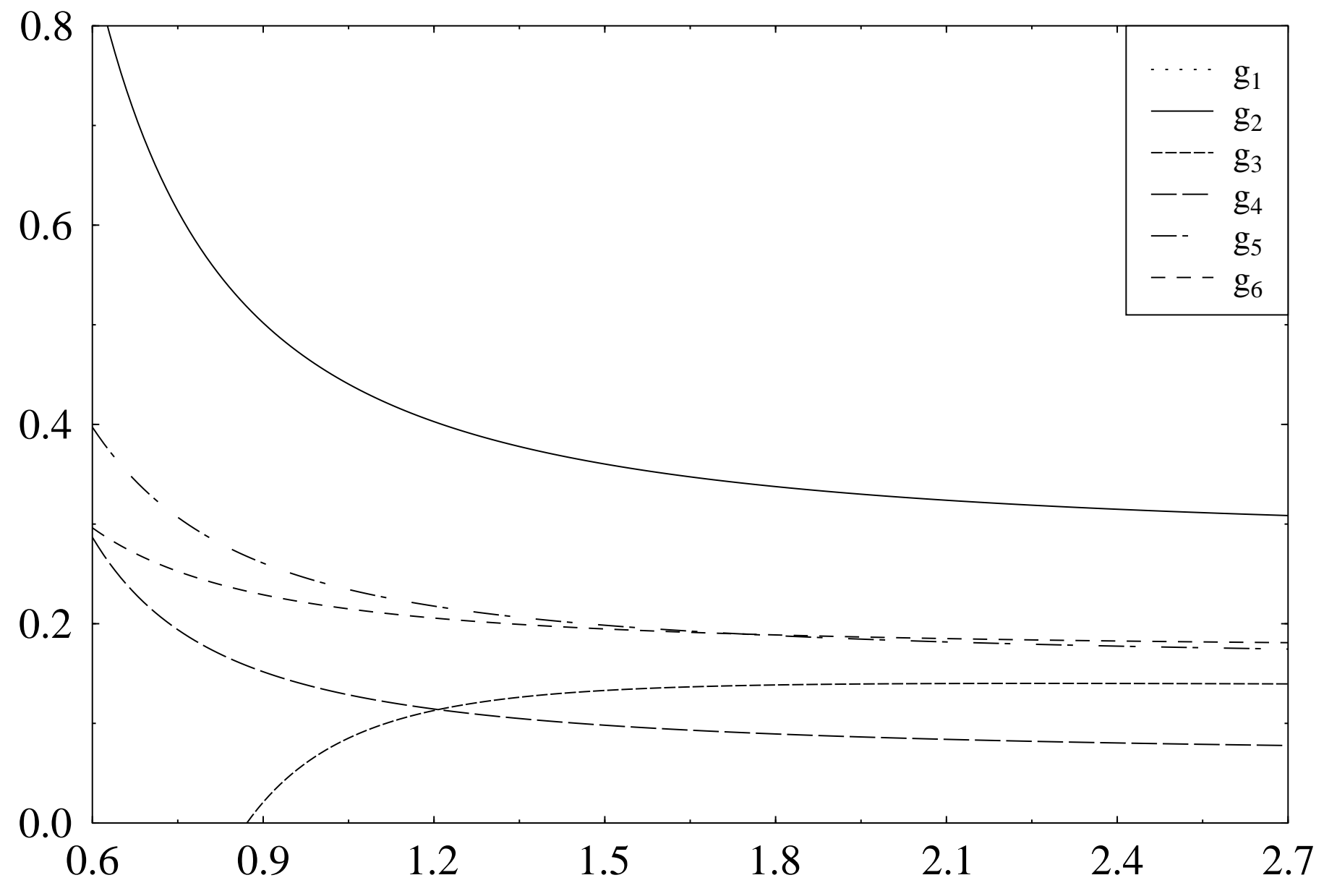

